\title{
Dynamic learning and memory, synaptic plasticity and neurogenesis: an update
}

\author{
Ales Stuchlik* \\ Institute of Physiology, Academy of Sciences of the Czech Republic, Prague, Czech Republic
}

\section{Edited by:}

Tomiki Sumiyoshi, National Center of

Neurology and Psychiatry, Japan

Reviewed by:

Phillip R. Zoladz, Ohio Northern

University, USA

Eddy A. Van Der Zee, University of

Groningen, Netherlands

*Correspondence:

Ales Stuchlik, Institute of Physiology,

Academy of Sciences of the Czech

Republic, Videnska 1803, 14220

Prague, Czech Republic

e-mail: stuchlik@biomed.cas.cz
Mammalian memory is the result of the interaction of millions of neurons in the brain and their coordinated activity. Candidate mechanisms for memory are synaptic plasticity changes, such as long-term potentiation (LTP). LTP is essentially an electrophysiological phenomenon manifested in hours-lasting increase on postsynaptic potentials after synapse tetanization. It is thought to ensure long-term changes in synaptic efficacy in distributed networks, leading to persistent changes in the behavioral patterns, actions and choices, which are often interpreted as the retention of information, i.e., memory. Interestingly, new neurons are born in the mammalian brain and adult hippocampal neurogenesis is proposed to provide a substrate for dynamic and flexible aspects of behavior such as pattern separation, prevention of interference, flexibility of behavior and memory resolution. This work provides a brief review on the memory and involvement of LTP and adult neurogenesis in memory phenomena.

Keywords: learning, memory, behavior, synaptic plasticity, adult neurogenesis, hippocampus

\section{INTRODUCTION}

The brain, with billions of cells' connections and plethora of cell types in mutual interactions, is one of the most complicated organs in the human body and attracts attention of both scientific researchers and the public. This is underlined by the fact that we do not know the pathophysiology of many devastating brain disorders, which often affect memory and cognition (such as schizophrenia, Alzheimer's disease etc.). Notably, the brain definitely does not work as an "elevated supervisor"; indeed, researchers working in neuroscience have now started to appreciate the "systems-level" understanding of the human body function in health and disease (Qureshi and Mehler, 2013).

Studying detailed physiology of any living system without evaluating its output (behavior), cannot give us enough information for understanding the function of the system. Vice versa, studying behavior without looking at the proximate mechanisms cannot provide sufficient insight, although striking exceptions from physiology exist such as ant navigation (e.g., Wohlgemuth et al., 2001), when scientists proceeded from studying behavioral outputs downstream to physiological mechanisms. Many scientists concerned with the integration of molecular and cellular views with systems-level and behavioral studies focus on the learning and memory. This particular function of nervous system in animals is well accessible by methods with different resolution (from spikes to molecules and cells to the whole organism).

In this review, I will try to provide a short update on integration of memory formation with a concept of synaptic plasticity (Hebb, 1949) (mainly long-term potentiation (LTP; Bliss and Lomo, 1973)) and adult neurogenesis in the dentate gyrus (DG) of the hippocampus (Altman, 1963; Altman and Das, 1965). My selection must obviously be subjective; there are excellent reviews on other issues related to this topic, such as the role of transcriptional factors and various kinases (Kandel, 2012; Xia and Storm, 2012). The rationale for selecting these subtopics settles on prevailing view on synaptic plasticity processes as a "hotspot" of current research into basic mechanisms of learning and memory (Glanzman, 2013). Focus on neurogenesis is based on the fact that despite many memory studies involve static settings, our world is endlessly dynamic and learning should be considered a highly dynamic process; neurogenesis may provide these flexible aspects of memory. These fields open ways for searching mechanisms of deficits in many neuropsychiatric disorders with enormous human and socioeconomic impact and suggest ways of novel causal therapies for depression, PTSD, Alzheimer's disease or schizophrenia (Voineskos et al., 2013). Scientists relate both LTP and neurogenesis to memory (Brown et al., 1990; Snyder et al., 2001), LTP as a candidate mechanism for longterm retention of information and hippocampal neurogenesis as a candidate mechanism for specific dynamic and flexible aspects of learning.

\section{LEARNING AND MEMORY}

Memory refers to a capability of virtually any animal to encode, store and retrieve information, to guide behavioral output. Learning is viewed as acquisition or encoding the information to memory. It is an excellent example of model system allowing for multi-level analysis (again, note close relation of memory deficits to these pathological conditions). Absence/presence of a memory trace (engram) is often presented or even defined as a change of a particular behavior (such as a rat with lesions to the hippocampus may get lost in a spatial maze), or then, as a change of ability to learn/remember. The term engram was first coined by Richard Semon, a German biologist (Semon, 1921). It usually 
refers to mechanisms (or tags) by which the memories are stored. The prevailing view today is that memory should leave physical (e.g., molecular) and often rather distributed changes in neuronal tissue (Moser and Moser, 1998; Frey and Frey, 2008).

Another well-accepted opinion is that there is nothing like "universal memory"; instead, multiple memory systems exist (Doeller et al., 2008; Lee et al., 2008; Schwabe, 2013) having their specific (partially competing but also shared) brain resources to fulfill their tasks (Squire, 2004). Temptating is a concept that memory may be stored (at least in mammals) in distributed changes in synaptic weights, which may modulate synchronization and grouping of firing of neuronal assemblies (Hebb, 1949; Harris et al., 2003). Further introspection of the engram and its nature also provokes many questions about stability, localization, time course of possible changes, and consolidation and transformation of a memory trace (reviewed in Dudai, 2004).

Importantly, complex and vulnerable mammalian memory types, i.e., declarative and spatial memory, depend on the medial temporal lobes (MTL) of the brain (reviewed in Eichenbaum, 2001). These are the hippocampus, subiculum and neighboring cortical areas such as entorhinal, perirhinal and postrhinal cortices (Amaral and Witter, 1989). Many scientist today are convinced that the hottest candidates for neural correlate of a longterm memory trace are long-term changes in synaptic strengths (Hebb, 1949), i.e., LTP and long-term depression (LTD). For the sake of simplicity, this minireview selects LTP out of the synaptic plasticity mechanisms, although there is a great evidence for LTD role in learning and memory as well (reviewed in Kemp and Manahan-Vaughan, 2007).

\section{LTP, PROTEIN KINASE Mzeta AND MEMORY}

Already in 1973, Terje Lomo a Timothy Bliss published a seminal study (Bliss and Lomo, 1973) showing that tetanization of specific pathway in the hippocampal formation of anesthetized rabbit resulted in significant increase in the excitatory postsynaptic potentials in postsynaptic cells, which further supported Hebb's theories (Hebb, 1949). Later on, this phenomenon was demonstrated in anesthetized and freely moving rats and mice. Today, LTP attracts many scientists, because it represents an intriguing but artificial model of long-term changes of the CNS function (more than 12,000 hits in PubMed on the term search). However, causal link between LTP and memory has been suspected but not settled for a long time, although supportive studies were provided earlier, in which interference with LTP affected learning and memory.

Multiple studies have demonstrated that interference with NMDA and AMPA receptor function (e.g., Steele and Morris, 1999; Bast et al., 2005) blocks certain phases of LTP and memory. Importance of NMDA receptors was documented especially in one-trial learning, such as in the delayed-matching to place version of the Morris water maze (MWM), a classical spatial task (Steele and Morris, 1999). Other studies used a different approach, i.e., tetanization of the majority of hippocampal synapses and subsequent testing in hippocampus-dependent task. Initially, it was shown that such LTP saturation disrupts subsequent spatial memory in the MWM probe trials (Moser and
Moser, 1999), but this effect has been found to be eliminated by non-spatial pretraining (Otnæss et al., 1999), suggesting that LTP induction may provide a mechanism for capturing the proper strategy in the task. Despite controversies, all these studies corroborated the hypothesis that LTP has some relation to learning and memory.

In 2006, two studies convincingly reported the link between LTP and learning and memory. Whitlock et al. (2006) examined the hypothesis that not only tetanization but also memory encoding per se may induce long-term plastic changes. The study showed that training rats to solve inhibitory avoidance, in which animal learns to avoid a preferred dark compartment punished by mild footshocks led to induction of the LTP in a subset of hippocampal synapses. Some synapses were unaffected, again supporting the concept of distributed memory trace. It is interesting to note that inhibitory avoidance learning, despite a simple paradigm, contains both operant and contextual fear conditioning component and involves highly coordinated recruitment of molecular and cellular machinery in the hippocampal formation (Izquierdo et al., 2002). The paper by Whitlock and colleagues contributed much to the notion that memory encoding may produce LTP in some synapses and that memory acquisition could be analogized to electrical tetanization of the synapse. Other studies have strongly corroborated this view (Cohen et al., 2011; Rodríguez-Durán et al., 2011; Kenney and Manahan-Vaughan, 2013).

Another paper in the same issue of Science (Pastalkova et al., 2006) focused on the maintenance phase of LTP as a candidate mechanism for memory storage, based on previous robust evidence from the laboratory of Todd Sacktor that an atypical form of protein kinase C (PKMzeta) is necessary and sufficient for maintenance phase of the LTP (Ling et al., 2002). The study employed so-called zeta-inhibiting peptide (ZIP), which was injected into hippocampi of rats that previously acquired the spatial active place avoidance task. Subsequently, memory retention was tested and it was found selectively impaired by ZIP injection. Interestingly, such microinjection failed to abolish novel learning in the same task, suggesting that PKMzeta erased previous memory trace but did not block encoding of novel information.

Subsequently, Shema et al. (2007) have shown that injection of ZIP into the insular cortex of the rat erased conditioned taste aversion, an evolutionary advantageous type of conditioning. This memory is traditionally measured by avoidance of ingestion of a food, the flavor of which had been associated previously with sickness (Buresová et al., 1979). Another study has corroborated these findings by extension to erasure of other types of memory, such as classical or instrumental conditioning (Serrano et al., 2008). Injection of ZIP also reduced the precision of the MWM representation in the probe trial, despite the rough localization of the goal was still present. Additional evidence on PKMzeta and memory storage came from a recent study (Shema et al., 2011), which showed that overexpression of PKMzeta in the insular region enhanced the conditioned taste aversion memory. Moreover, Pauli et al. (2012) has revealed that blockade of PKMzeta also has an effect in the striatum, affecting instrumental response selection and habits. 
However, Volk et al. (2013) have recently generated constitutive and conditional PKMzeta-knockout mice and detected no impairment of either LTP maintenance or hippocampusdependent memory (Volk et al., 2013). Since the previous reports sometimes used pharmacological blockade of enzyme by ZIP, Volk et al. also applied ZIP to their transgenic mice lacking PKMzeta and detected LTP suppression. This suggests ZIP targets other enzymes required for LTP. Analogous results have been found by Lee et al. (2013) in PKMzeta-null mice. The role of PKMzeta has therefore been questioned (Kwapis and Helmstetter, 2013).

Interestingly, another form of PKC named iota (Selbie et al., 1993) was suggested to compensate for deficient PKMzeta in these experiments, suggesting that more diverse cascade can provide basis for memory maintenance rather than a single "memory molecule" (Glanzman, 2013). In any case, scientists appear to be on the track of interesting discovery of how our vivid everyday memories relate to molecular and cellular brain processes.

\section{ADULT NEUROGENESIS IN THE DENTATE GYRUS AND MEMORY}

Importantly, the hippocampus, specifically the subgranular zone of the DG, is one of two sites of neurogenesis in the adult brain (Altman, 1963; Altman and Das, 1965). Some of the dividing neural progenitor cells survive, differentiate into neurons and incorporate into the hippocampal network. The physiological, especially the behavioral role of adult neurogenesis is a subject of controversies until today. Newly born neurons in the DG are proposed to facilitate learning in the hippocampus by separating overlapping patterns in hippocampal inputs, thus ensuring formation of distinct representations.

Enhancing adult neurogenesis in the DG was found to suffice for improvement of pattern separation (Sahay et al., 2011) and another recent study (Nakashiba et al., 2012) suggested that newly-born hyper-excitable neurons may participate preferentially in pattern separation, while older adult granule neurons provide mainly pattern completion (a complementary process which allows adding missing features into incomplete hippocampal representations). This study did not focus directly on the autoassociative network in CA3, but in light of paper by Rolls (2013), I propose that strong, "consolidated" synapses between older granule neurons via mossy fibers to CA3 may provide a significant contribution to pattern completion. It should be noted that a precise balance between pattern completion and pattern separation is probably one if the functions of CA3 upstream region innervated by mossy fibers from dentate granule cells, which mediates a proper hippocampus function and such interplay may be disrupted in memory disorders (Hanson and Madison, 2010).

Another branch of research on the functional role of adult neurogenesis has proposed that adult neurogenesis in the hippocampus may also ensure prevention of interference of new memories with old ones (Wiskott et al., 2006). A study using olfactory memory task demanding interference resolution has also supported such role for new granule neurons in the DG (Luu et al., 2012). A recent study by Gordon Winocur et al. have strongly supported this prediction (Winocur et al., 2012) using a visual discrimination task under conditions of low or high interference. A very recent study by Déry et al. (2013) confirmed such role of neurogenesis even in humans and shown a positive impact of voluntary exercise and adverse effects of depression. Recently, the role of DG adult neurogenesis has been extended to increase "memory resolution" so that cooperation between newly born, hyperexcitable granule cells and older neurons that code sparsely for salient features increases the amount of detail encoded in hippocampal memories (Aimone et al., 2011).

Studies also showed that hippocampal neurogenesis promotes behavioral flexibility in mice. A study by Garthe et al. (2009) demonstrated the subtle but significant effects of neurogenesis ablation. Using chronic treatment with a cytostatic temozolomide and efficient recovery protocol, the memory differences between mice with and without adult neuronal proliferation were revealed in the MWM not by traditional measures such as distance to reach the platform, but by evaluating spatial/non-spatial strategies possessed in the maze. Robust evidence on behavioral flexibility account of neurogenesis has been provided by Burghardt et al. (2012), who showed that neurogenesis ablation in mice led to impairments of reversal learning in active place avoidance task (reviewed by Stuchlik et al., 2013). It also disrupted flexible incorporation of second reference frame (second to-be-avoided place, i.e., two-frame place avoidance; reviewed in Stuchlik et al., 2013). The deficit could not be explained by alteration of memory extinction, or with inability to acquire new memory in a novel environment; these functions were spared in both groups. Interestingly, the flexibility impairment was accompanied by immediate-early gene $\operatorname{Arc}$ up-regulation, suggesting effects on excitability and plasticity of the hippocampal network (Pevzner et al., 2012).

Recently, time-limited role of new, hyper-excitable granule neurons in the DG in hippocampus-dependent memory has been shown by optogenetic approach ( $\mathrm{Gu}$ et al., 2012). This study showed that newly born cells form functional synapses on pyramidal neurons of CA3 region from 2 weeks after their birth reaching a stable state at 4 weeks. Newborn neurons at this age were more plastic than neurons at other stages. The study also showed that switching off this cell type of 4-week-old cells after encoding disrupted retrieval of hippocampal memory. This suggests that these 4 weeks represent a functional time window for adult-born neurons in hippocampus-dependent memory retrieval (Gu et al., 2012). Using multiple high-resolution methods, a study of Ikrar et al. (2013) clearly documented that blocking adult neurogenesis increased excitability in the DG networks while its enhancement has reduced it, and also pointed to an important role of inhibitory GABAergic interneurons (Ikrar et al., 2013).

Based on this, we propose that neurogenesis in the hippocampus might underlie so-called behavioral separation, which we define as selective recruitment of distinct and dissociable hippocampus-functions such as spatial representation and cognitive coordination (Kubík and Fenton, 2005; Wesierska et al., 2005). Such role might be shown by selective neurogenesis ablation and testing in massed/alternate protocols using the specific behavioral tasks (MWM, Morris, 1981) and active place avoidance on Carousel (Stuchlik et al., 2013). Additionally, newly 
born neurons may contribute to coping with dynamic, changing aspects of memory (such as with moving goals) in accordance with the evolutionary hypothesis of neurogenesis role (Kempermann, 2012).

\section{SHORT NOTES ON SOME OTHER FACTORS AFFECTING LTP, NEUROGENESIS AND MEMORY}

There are additional factors affecting memory, LTP, and neurogenesis. For instance, a recent study on human glial cells implanted into mouse hippocampus documented enhanced LTP, suggesting strong functional role of this cell type in the brain (Zhang and Barres, 2013). Neurogenesis is also significantly affected by ageing, stress and disease but contrarily enhanced e.g., by enriched environment (Kempermann et al., 1997) and physical exercise (van Praag et al., 1999). Generally, all these phenomena in parallel affect memory as well (Nilsson et al., 1999; Lithfous et al., 2013). An intriguing study by Van der Borght et al. (2007) showed beneficial effects of physical exercise and dietary restriction on spatial T-maze performance and surprisingly, T-maze learning and reversal training actually led to reduced neurogenesis, suggesting optimal balance of these processes for proper memory maintenance.

Of high importance is the role of sleep in memory (McCoy et al., 2013). It has been shown that during both non-REM and REM sleep cellular processes may take part, subserving memory (Benington and Frank, 2003). A factual necessity of sleep for forming and consolidation of memories has emerged recently (Prince et al., 2014). Sleep is also related to neurogenesis (Mueller et al., 2013) as well as to LTP (Kim et al., 2005). From a pharmacological point of view, a recent study have shown that anti-diabetes drug metformin might offer a promising way of increasing adult neurogenesis and memory function (Wang et al., 2012) that is impaired in several neuropsychiatric disorders.

\section{CONCLUSIONS}

Much evidence converges on the view that learning and memory, synaptic plasticity and neurogenesis are inter-related phenomena. Specifically, the latter two are considered to provide substrate for specific aspects of learning and memory function. LTP maintenance probably underlies memory retention and its inhibition erases memory. Encoding of a memory trace induces LTP in subset of hippocampal synapses. Neurogenesis underlies specific dynamic and flexible features of learning and memory phenomena in the precisely regulated and time-restricted manner. Nowadays and in the future, collaborative and multidisciplinary efforts involving optogenetics, transgenes, in vivo patch-clamp etc. will bring significant insight into mechanisms of memory.

\section{ACKNOWLEDGMENTS}

This work was supported by GACR grant 14-03627S, Academic project M200111204, and institutional support RVO67985823. We thank all colleagues from the laboratory for their support, to Prof. P. Mares for critical reading and comments on the manuscript and to T. Hua and P. Luketic for proofreading.

\section{REFERENCES}

Aimone, J. B., Deng, W., and Gage, F. H. (2011). Resolving new memories: a critical look at the dentate gyrus, adult neurogenesis and pattern separation. Neuron 70, 589-596. doi: 10.1016/j.neuron.2011.05.010

Altman, J. (1963). Autoradiographic investigation of cell proliferation in the brains of rats and cats. Anat. Rec. 145, 573-591. doi: 10.1002/ar.1091450409

Altman, J., and Das, G. D. (1965). Autoradiographic and histological evidence of postnatal hippocampal neurogenesis in rats. J. Comp. Neurol. 124, 319-335. doi: $10.1002 /$ cne. 901240303

Amaral, D. G., and Witter, M. P. (1989). The three-dimensional organization of the hippocampal formation: a review of anatomical data. Neuroscience 31, 571-591. doi: 10.1016/0306-4522(89)90424-7

Bast, T., da Silva, B. M., and Morris, R. G. M. (2005). Distinct contributions of hippocampal NMDA and AMPA receptors to encoding and retrieval of one-trial place memory. J. Neurosci. 25, 5845-5856. doi: 10.1523/jneurosci.0698-05.2005

Benington, J. H., and Frank, M. G. (2003). Cellular and molecular connections between sleep and synaptic plasticity. Prog. Neurobiol. 69, 71-101. doi: 10. 1016/S0301-0082(03)00018-2

Bliss, T. V., and Lomo, T. (1973). Long-lasting potentiation of synaptic transmission in the dentate area of the anaesthetized rabbit following stimulation of the perforant path. J. Physiol. 232, 331-356.

Brown, T. H., Kairiss, E. W., and Keenan, C. L. (1990). Hebbian synapses: biophysical mechanisms and algorithms. Annu. Rev. Neurosci. 13, 475-511. doi: 10. 1146/annurev.neuro.13.1.475

Buresová, O., Aleksanyan, Z. A., and Bures, J. (1979). Electrophysiological analysis of retrieval of conditioned taste aversion in rats. Unit activity changes in critical brain regions. Physiol. Bohemoslov. 28, 525-536.

Burghardt, N. S., Park, E. H., Hen, R., and Fenton, A. A. (2012). Adult-born hippocampal neurons promote cognitive flexibility in mice. Hippocampus 22, 1795-1808. doi: 10.1002/hipo.22013

Cohen, Y., Avramoav, S., Barkai, E., and Maroun, M. (2011). Olfactory learninginduced enhancement of the predisposition for LTP induction. Learn. Mem. 18, 594-597. doi: 10.1101/lm.2231911

Déry, N., Pilgrim, M., Gibala, M., Gillen, J., Wojtowicz, J. M., Macqueen, G., et al. (2013). Adult hippocampal neurogenesis reduces memory interference in humans: opposing effects of aerobic exercise and depression. Front. Neurosci. 7:66. doi: 10.3389/fnins.2013.00066

Doeller, C. F., King, J. A., and Burgess, N. (2008). Parallel striatal and hippocampal systems for landmarks and boundaries in spatial memory. Proc. Natl. Acad. Sci. U S A 105, 5915-5920. doi: 10.1073/pnas.0801489105

Dudai, Y. (2004). The neurobiology of consolidations, or, how stable is the engram? Annu. Rev. Psychol. 55, 51-86. doi: 10.1146/annurev.psych.55.090902.142050

Eichenbaum, H. (2001). The hippocampus and declarative memory: cognitive mechanisms and neural codes. Behav. Brain Res. 127, 199-207. doi: 10. 1016/s0166-4328(01)00365-5

Frey, S., and Frey, J. U. (2008). 'Synaptic tagging' and 'cross-tagging' and related associative reinforcement processes of functional plasticity as the cellular basis for memory formation. Prog. Brain Res. 169, 117-143. doi: 10.1016/s00796123(07)00007-6

Garthe, A., Behr, J., and Kempermann, G. (2009). Adult-generated hippocampal neurons allow the flexible use of spatially precise learning strategies. PLoS One 4:e5464. doi: 10.1371/journal.pone.0005464

Glanzman, D. L. (2013). PKM and the maintenance of memory. F1000 Biol. Rep. 5:4. doi: 10.3410/B5-4

Gu, Y., Arruda-Carvalho, M., Wang, J., Janoschka, S. R., Josselyn, S. A., Frankland, P. W., et al. (2012). Optical controlling reveals time-dependent roles for adultborn dentate granule cells. Nat. Neurosci. 15, 1700-1706. doi: 10.1038/nn. 3260

Hanson, J. E., and Madison, D. V. (2010). Imbalanced pattern completion vs. separation in cognitive disease: network simulations of synaptic pathologies predict a personalized therapeutics strategy. BMC Neurosci. 11:96. doi: 10. 1186/1471-2202-11-96

Harris, K. D., Csicsvari, J., Hirase, H., and Dragoi, G. (2003). Organization of cell assemblies in the hippocampus. Nature 424, 552-556. doi: 10.1038/nature 01834

Hebb, D. (1949). The Organization of Behavior. New York: Wiley.

Ikrar, T., Guo, N., He, K., Besnard, A., Levinson, S., Hill, A., et al. (2013). Adult neurogenesis modifies excitability of the dentate gyrus. Front. Neural Circuits 7:204. doi: 10.3389/fncir.2013.00204 
Izquierdo, L. A., Barros, D. M., Vianna, M. R., Coitinho, A., deDavid e Silva, T., Choi, H., et al. (2002). Molecular pharmacological dissection of short- and long-term memory. Cell. Mol. Neurobiol. 22, 269-287. doi: 10.1023/A:1020715 800956

Kandel, E. R. (2012). The molecular biology of memory: cAMP, PKA, CRE, CREB1, CREB-2 and CPEB. Mol. Brain 5:14. doi: 10.1186/1756-6606-5-14

Kemp, A., and Manahan-Vaughan, D. (2007). Hippocampal long-term depression: master or minion in declarative memory processes? Trends Neurosci. 30, 111118. doi: 10.1016/j.tins.2007.01.002

Kempermann, G. (2012). New neurons for 'survival of the fittest'. Nat. Rev. Neurosci. 13, 727-736. doi: 10.1038/nrn3319

Kempermann, G., Kuhn, H. G., and Gage, F. H. (1997). More hippocampal neurons in adult mice living in an enriched environment. Nature 386, 493-495. doi: 10. 1038/386493a0

Kenney, J., and Manahan-Vaughan, D. (2013). Learning-facilitated synaptic plasticity occurs in the intermediate hippocampus in association with spatial learning. Front. Synaptic Neurosci. 29:10. doi: 10.3389/fnsyn.2013.00010

Kim, E. Y., Mahmoud, G. S., and Grover, L. M. (2005). REM sleep deprivation inhibits LTP in vivo in area CA1 of rat hippocampus. Neurosci. Lett. 388, 163167. doi: 10.1016/j.neulet.2005.06.057

Kubík, S., and Fenton, A. A. (2005). Behavioral evidence that segregation and representation are dissociable hippocampal functions. J. Neurosci. 25, 92059212. doi: 10.1523/jneurosci.1707-05.2005

Kwapis, J. L., and Helmstetter, F. J. (2013). Does PKM(zeta) maintain memory? Brain Res. Bull. doi: 10.1016/j.brainresbull.2013.09.005. [Epub ahead of print].

Lee, A. M., Kanter, B. R., Wang, D., Lim, J. P., Zou, M. E., Qiu, C., et al. (2013). Prkcz null mice show normal learning and memory. Nature 493, 416-419. doi: 10. 1038/nature11803

Lee, A. S., Duman, R. S., and Pittenger, C. (2008). A double dissociation revealing bidirectional competition between striatum and hippocampus during learning. Proc. Natl. Acad. Sci. U S A 105, 17163-17168. doi: 10.1073/pnas.0807749105

Ling, D. S. F., Benardo, L. S., Serrano, P. A., Blace, N., Kelly, M. T., Crary, J. F., et al. (2002). Protein kinase Mzeta is necessary and sufficient for LTP maintenance. Nat. Neurosci. 5, 295-296. doi: 10.1038/nn829

Lithfous, S., Dufour, A., and Despres, O. (2013). Spatial navigation in normal aging and the prodromal stage of Alzheimer's disease: insights from imaging and behavioral studies. Ageing Res. Rev. 12, 201-213. doi: 10.1016/j.arr.2012. 04.007

Luu, P., Sill, O. C., Gao, L., Becker, S., Wojtowicz, J. M., and Smith, D. M. (2012). The role of adult hippocampal neurogenesis in reducing interference. Behav. Neurosci. 126, 381-391. doi: 10.1037/a0028252

McCoy, J. G., Christie, M. A., Kim, Y., Brennan, R., Poeta, D. L., McCarley, R. W., et al. (2013). Chronic sleep restriction impairs spatial memory in rats. Neuroreport 24, 91-95. doi: 10.1097/wnr.0b013e32835cd97a

Morris, R. G. M. (1981). Spatial localization does not require presence of local cues. Learn. Mot. 260, 239-260. doi: 10.1016/0023-9690(81)90020-5

Moser, E. I., and Moser, M. B. (1999). Is learning blocked by saturation of synaptic weights in the hippocampus? Neurosci. Biobehav. Rev. 23, 661-672. doi: 10. 1016/s0149-7634(98)00060-8

Moser, M., and Moser, E. I. (1998). Distributed encoding and retrieval of spatial memory in the hippocampus. J. Neurosci. 18, 7535-7542.

Mueller, A. D., Meerlo, P., McGinty, D., and Mistlberger, R. E. (2013). Sleep and adult neurogenesis: implications for cognition and mood. Curr. Top. Behav. Neurosci. doi: 10.1007/7854_2013_251. [Epub ahead of Print].

Nakashiba, T., Cushman, J. D., Pelkeym, K. A., Renaudineau, S., Buhl, D. L., McHugh, T. J., et al. (2012). Young dentate granule cells mediate pattern separation, whereas old granule cells facilitate pattern completion. Cell 149, 188201. doi: 10.1016/j.cell.2012.01.046

Nilsson, M., Perfilieva, E., Johansson, U., Orwar, O., and Eriksson, P. S. (1999). Enriched environment increases neurogenesis in the adult rat dentate gyrus and improves spatial memory. J. Neurobiol. 39, 569-578. doi: 10.1002/(sici)10974695(19990615)39:4<569::aid-neu10>3.0.co;2-f

Otnæss, M. K., Brun, V. H., Moser, M. B., and Moser, E. I. (1999). Pretraining prevents spatial learning impairment after saturation of hippocampal long-term potentiation. J. Neurosci. 19, RC49.

Pastalkova, E., Serrano, P., Pinkhasova, D., Wallace, E., Fenton, A., and Sacktor, T. C. (2006). Storage of spatial information by the maintenance mechanism of LTP. Science 313, 1141-1144. doi: 10.1126/science.1128657
Pauli, W. M., Clark, A. D., Guenther, H. J., O’Reilly, R. C., and Rudy, J. W. (2012). Inhibiting $\mathrm{PKM} \zeta$ reveals dorsal lateral and dorsal medial striatum store the different memories needed to support adaptive behavior. Learn. Mem. 19, $307-$ 314. doi: 10.1101/lm.025148.111

Pevzner, A., Miyashita, T., Schiffman, A. J., and Guzowski, J. F. (2012). Temporal dynamics of Arc gene induction in hippocampus: relationship to context memory formation. Neurobiol. Learn. Mem. 97, 313-320. doi: 10.1016/j.nlm.2012. 02.004

Prince, T. M., Wimmer, M., Choi, J., Havekes, R., Aton, S., and Abel, T. (2014). Sleep deprivation during a specific 3-hour time window post-training impairs hippocampal synaptic plasticity and memory. Neurobiol. Learn. Mem. 109, 122 130. doi: 10.1016/j.nlm.2013.11.021

Qureshi, I. A., and Mehler, M. F. (2013). Towards a "systems"-level understanding of the nervous system and its disorders. Trends Neurosci. 36, 674-684. doi: 10 . 1016/j.tins.2013.07.003

Rodríguez-Durán, L. F., Castillo, D. V., Moguel-González, M., and Escobar, M. L. (2011). Conditioned taste aversion modifies persistently the subsequent induction of neocortical long-term potentiation in vivo. Neurobiol. Learn. Mem. 95, 519-526. doi: 10.1016/j.nlm.2011.03.003

Rolls, E. T. (2013). The mechanisms for pattern completion and pattern separation in the hippocampus. Front. Syst. Neurosci. 7:74. doi: 10.3389/fnsys.2013. 00074

Sahay, A., Scobie, K. N., Hill, A. S., O’Carroll, C. M., Kheirbek, M. A., Burghardt, N. S., et al. (2011). Increasing adult hippocampal neurogenesis is sufficient to improve pattern separation. Nature 472, 466-470. doi: 10.1038/nature09817

Schwabe, L. (2013). Stress and the engagement of multiple memory systems: integration of animal and human studies. Hippocampus 23, 1035-1043. doi: 10. 1002/hipo. 22175

Selbie, L. A., Schmitz-Peiffer, C., Sheng, Y., and Biden, T. J. (1993). Molecular cloning and characterization of PKC iota, an atypical isoform of protein kinase C derived from insulin-secreting cells. J. Biol. Chem. 268, 24296-24302.

Semon, R. (1921). The Mneme. London: Allen and Unwin.

Serrano, P., Friedman, E. L., Kenney, J., Taubenfeld, S. M., Zimmerman, J. M., Hanna, J., et al. (2008). PKMzeta maintains spatial, instrumental, and classically conditioned long-term memories. PLoS Biol. 6:60318. doi: 10.1371/journal. pbio. 0060318

Shema, R., Haramati, S., Ron, S., Hazvi, S., Chen, A., Sacktor, T. C., et al. (2011). Enhancement of consolidated long-term memory by overexpression of protein kinase Mzeta in the neocortex. Science 331, 1207-1210. doi: 10.1126/science. 1200215

Shema, R., Sacktor, T. C., and Dudai, Y. (2007). Rapid erasure of long-term memory associations in the cortex by an inhibitor of PKM zeta. Science 317, 951-953. doi: $10.1126 /$ science. 1144334

Snyder, J. S., Kee, N., and Wojtowicz, J. M. (2001). Effects of adult neurogenesis on synaptic plasticity in the rat dentate gyrus. J. Neurophysiol. 85, 2423-2431.

Squire, L. R. (2004). Memory systems of the brain: a brief history and current perspective. Neurobiol. Learn. Mem. 82, 171-177. doi: 10.1016/j.nlm.2004. 06.005

Steele, R. J., and Morris, R. G. (1999). Delay-dependent impairment of a matching-to-place task with chronic and intrahippocampal infusion of the NMDA-antagonist D-AP5. Hippocampus 9, 118-136. doi: 10.1002/(sici)10981063(1999)9:2<118::aid-hipo4>3.0.co;2-8

Stuchlik, A., Petrasek, T., Prokopova, I., Holubova, K., Hatalova, H., Vales, K., et al. (2013). Place avoidance tasks as tools in the behavioral neuroscience of learning and memory. Physiol. Res. 62, S1-S19.

Van der Borght, K., Havekes, R., Bos, T., Eggen, B. J., and Van der Zee, E. A. (2007). Exercise improves memory acquisition and retrieval in the Y-maze task: relationship with hippocampal neurogenesis. Behav. Neurosci. 121, 324-334. doi: 10.1037/0735-7044.121.2.324

van Praag, H., Christie, B. R., Sejnowski, T. J., and Gage, F. H. (1999). Running enhances neurogenesis, learning, and long-term potentiation in mice. Proc. Natl. Acad. Sci. U S A 96, 13427-13431. doi: 10.1073/pnas.96.23.13427

Voineskos, D., Rogasch, N. C., Rajji, T. K., Fitzgerald, P. B., and Daskalakis, Z. J. (2013). A review of evidence linking disrupted neural plasticity to schizophrenia. Can. J. Psychiatry 58, 86-92.

Volk, L. J., Bachman, J. L., Johnson, R., Yu, Y., and Huganir, R. L. (2013). PKM- $\zeta$ is not required for hippocampal synaptic plasticity, learning, and memory. Nature 493, 420-423. doi: 10.1038/nature11802 
Wang, J., Gallagher, D., DeVito, L. M., Cancino, G. I., Tsui, D., He, L., et al. (2012). Metformin activates an atypical PKC-CBP pathway to promote neurogenesis and enhance spatial memory formation. Cell Stem Cell 11, 23-35. doi: 10.1016/j. stem.2012.03.016

Wesierska, M., Dockery, C., and Fenton, A. A. (2005). Beyond memory, navigation, and inhibition: behavioral evidence for hippocampus-dependent cognitive coordination in the rat. J. Neurosci. 25, 2413-2419. doi: 10.1523/jneurosci.396204.2005

Whitlock, J. R., Heynen, A. J., Shuler, M. G., and Bear, M. F. (2006). Learning induces long-term potentiation in the hippocampus. Science 313, 1093-1097. doi: 10.1126/science. 1128134

Winocur, G., Becker, S., Luu, P., Rosenzweig, S., and Wojtowicz, J. M. (2012). Adult hippocampal neurogenesis and memory interference. Behav. Brain Res. 227, 464-469. doi: 10.1016/j.bbr.2011.05.032

Wiskott, L., Rasch, M. J., and Kempermann, G. (2006). A functional hypothesis for adult hippocampal neurogenesis: avoidance of catastrophic interference in the dentate gyrus. Hippocampus 16, 329-343. doi: 10.1002/hipo. 20167

Wohlgemuth, S., Ronacher, B., and Wehner, R. (2001). Ant odometry in the third dimension. Nature 411, 795-798. doi: 10.1038/35081069
Xia, Z., and Storm, D. R. (2012). Role of signal transduction crosstalk between adenylyl cyclase and MAP kinase in hippocampus-dependent memory. Learn. Mem. 19, 369-374. doi: 10.1101/lm.027128.112

Zhang, Y., and Barres, B. A. (2013). A smarter mouse with human astrocytes. Bioessays 35, 876-880. doi: 10.1002/bies.201300070

Conflict of Interest Statement: The author declares that the research was conducted in the absence of any commercial or financial relationships that could be construed as a potential conflict of interest.

Received: 30 January 2014; accepted: 13 March 2014; published online: 01 April 2014. Citation: Stuchlik A (2014) Dynamic learning and memory, synaptic plasticity and neurogenesis: an update. Front. Behav. Neurosci. 8:106. doi: 10.3389/fnbeh.2014.00106 This article was submitted to the journal Frontiers in Behavioral Neuroscience.

Copyright (C) 2014 Stuchlik. This is an open-access article distributed under the terms of the Creative Commons Attribution License (CC BY). The use, distribution or reproduction in other forums is permitted, provided the original author(s) or licensor are credited and that the original publication in this journal is cited, in accordance with accepted academic practice. No use, distribution or reproduction is permitted which does not comply with these terms. 